\title{
PREVALENCE OF PLAYING RELATED MUSCULOSKELETAL DISORDERS IN MUSICIANS
}

\author{
Krupa Gohil ${ }^{*}$, Megha Sheth, Neeta Vyas \\ S.B.B College of Physiotherapy, V.S Hospital Campus, Ellis Bridge, Ahmedabad-380006.
}

\begin{abstract}
Introduction: Musculoskeletal disorders in musicians include pain, weakness, numbness, tingling, or other symptoms that interfere with their ability to play their instrument at the level they are accustomed to. Many studies find a high prevalence of work-related musculoskeletal disorders in musicians, ranging from $73.4 \%$ to $87.7 \%$. String players have the highest prevalence of musculoskeletal problems. There are very few studies in India and none in Gujarat, thus there is a need to study prevalence of musculoskeletal disorders in musicians.

Method: A survey study using convenience sampling technique was done from the music schools of Ahmedabad with 50 subjects. The subjects aged 10 to 40 years, both males and females, with use of instruments more than 3 months were included in the study. Subjects were asked to fill questionnaire concerning demographic data, information about music and Cornell's musculoskeletal disorders questionnaire.
\end{abstract}

Result: 9 out of 50(18\%) musicians had musculoskeletal disorders. Cornell's musculoskeletal disorders questionnaire score ranged from 1.5-57 with mean 10.61 \pm 17.81 .

Conclusion: $18 \%$ musicians were found to have musculoskeletal disorders.

Key words: Musculoskeletal disorders, Musicians, Cornell's musculoskeletal disorders questionnaire

\section{INTRODUCTION}

Musculoskeletal disorders in musicians includepain, weakness, numbness, tingling or other symptoms that interfere with their ability to play their instrument at the level they are accustomed to.[1]Work-related musculoskeletal disorders cause pain, disability and loss of employment for many workers, including musicians.[2]

A high prevalence of work-related musculoskeletal disorders has been found in musicians, ranging from $73.4 \%$ to $87.7 \%$.[3] Lee H S examined the various positions and movements of the upper extremities which cause pain in musicians: 1) basic postures for holding instruments, 2) movements of left upper extremity: fingering, forearm posture, high position and vibrato, 3) movements of right upper extremity: bowing, bow angles, pizzicato and other bowing techniques. These isotonic and isometric movements can lead to musculoskeletal problems in musicians.[3] They also reviewed orthopedic disorders that are specific to string players: overuse syndrome, muscle-tendon syndrome, focal dystonia, hypermobility syndrome, and compressive neuropathy.[3] According to Berque $P$, Musicians reported their loss of facility in

*Corresponding author:

Email: krupagohil5@gmail.com

http://dx.doi.org/10.20530/IJTA $32 \quad$ 100-103

ISSN 2320-138X (c) 2016 playing in terms of: loss of speed, loss of control of major motions, loss of control of fine motions, loss of power and loss of finger span.[1]

There are very few studies in India and none in Gujarat on musicians. So the study aimed to find the prevalence of playing related musculoskeletal disorders (PRMD) in musicians in Ahmedabad, the severity of PRMD and the correlation between the usage of instrument per week (in hours)\& Cornell's musculoskeletal disorders questionnaire score and the total period of usage of the instrument (in months) \& Cornell's musculoskeletal disorders questionnaire score.

\section{METHODOLOGY}

A cross sectional survey study having convenience sampling was conducted in Ahmedabad community. Fifty male and female musicians aged 10 to 40 years who have been using their instruments for more than 3 months were included in the study. The musicians who had history of trauma and history of congenital orthopaedic conditions and congenital neurological conditions were excluded from the study.

The nature and purpose of study was explained and informed consent was obtained from participants prior to the study. The musicians were asked to fill questionnaire concerning demographic data and information about music instrument usage. The musicians who had pain 


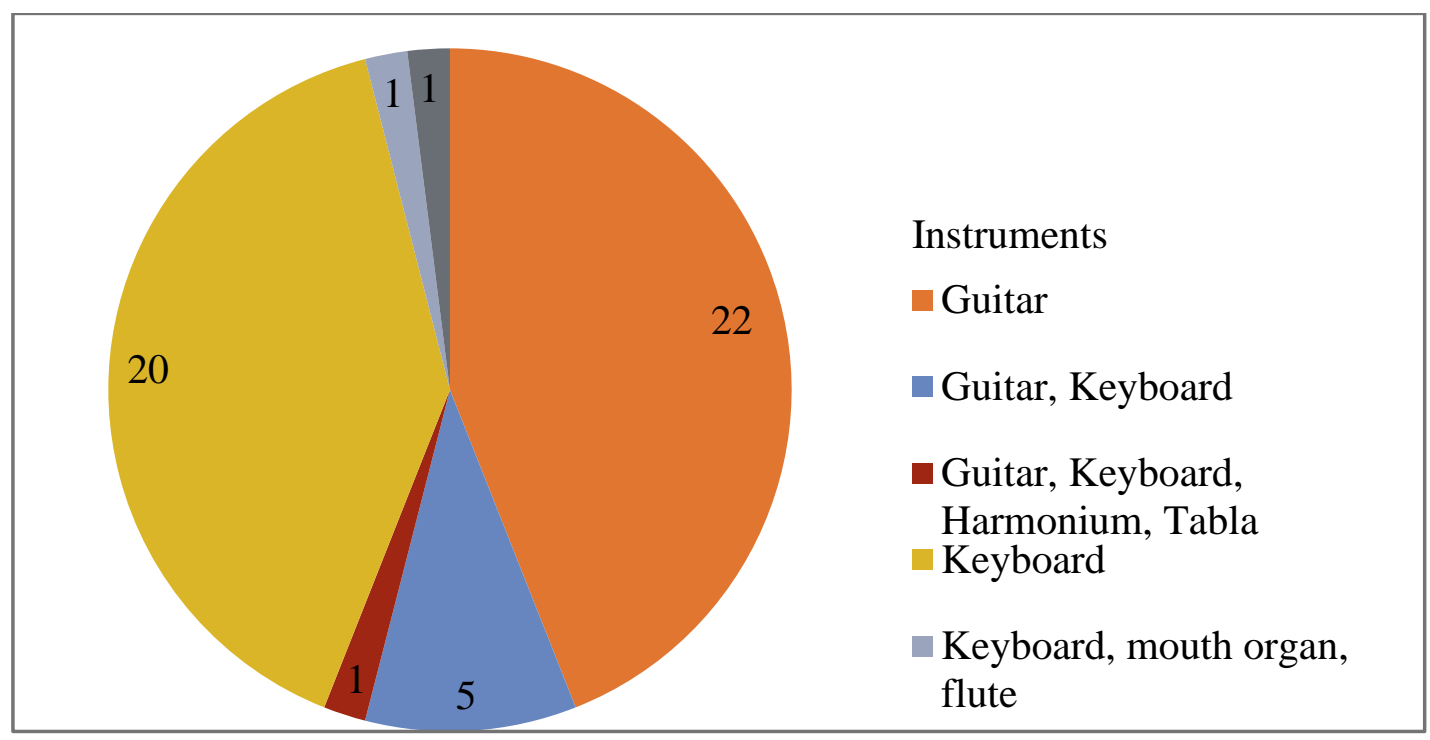

Figure 1: Types of instruments

and discomfort were asked to fill the Cornell's musculoskeletal disorders questionnaire (CMDQ).

Statistical analysis was done for correlation between the usage of instrument per week (in hours) \& the Cornell's musculoskeletal disorders questionnaire score and the total period of usage of the instrument (in months) \& the Cornell's musculoskeletal disorders questionnaire score. The level of significance kept at $5 \%$.

\section{RESULT}

Figure 1 shows the instruments used by the musicians. As seen in the Figure, musicians who were using guitar and keyboard were found to be more in number, i.e. 47 out of 50 . Nine musicians out of 50 were found to have musculoskeletal disorders. Out of them: 7 had wrist pain( 77\%), 5 had neck pain(55\%), 3 had forearm pain(33\%), 1 had upper back pain(11\%) and 1 had leg pain(11\%).

Correlation between the usage of instrument per week (in hours) \& CMDQ score was done by using non parametric Spearman's test. Correlation co-efficient was -0.507 as shown in Figure 2 . Correlation between the total period of usage of the instrument (in months) \& CMDQ score was done by using non parametric Spearman's test and itscorrelation co-efficient was -0.525 as shown in Figure 3.

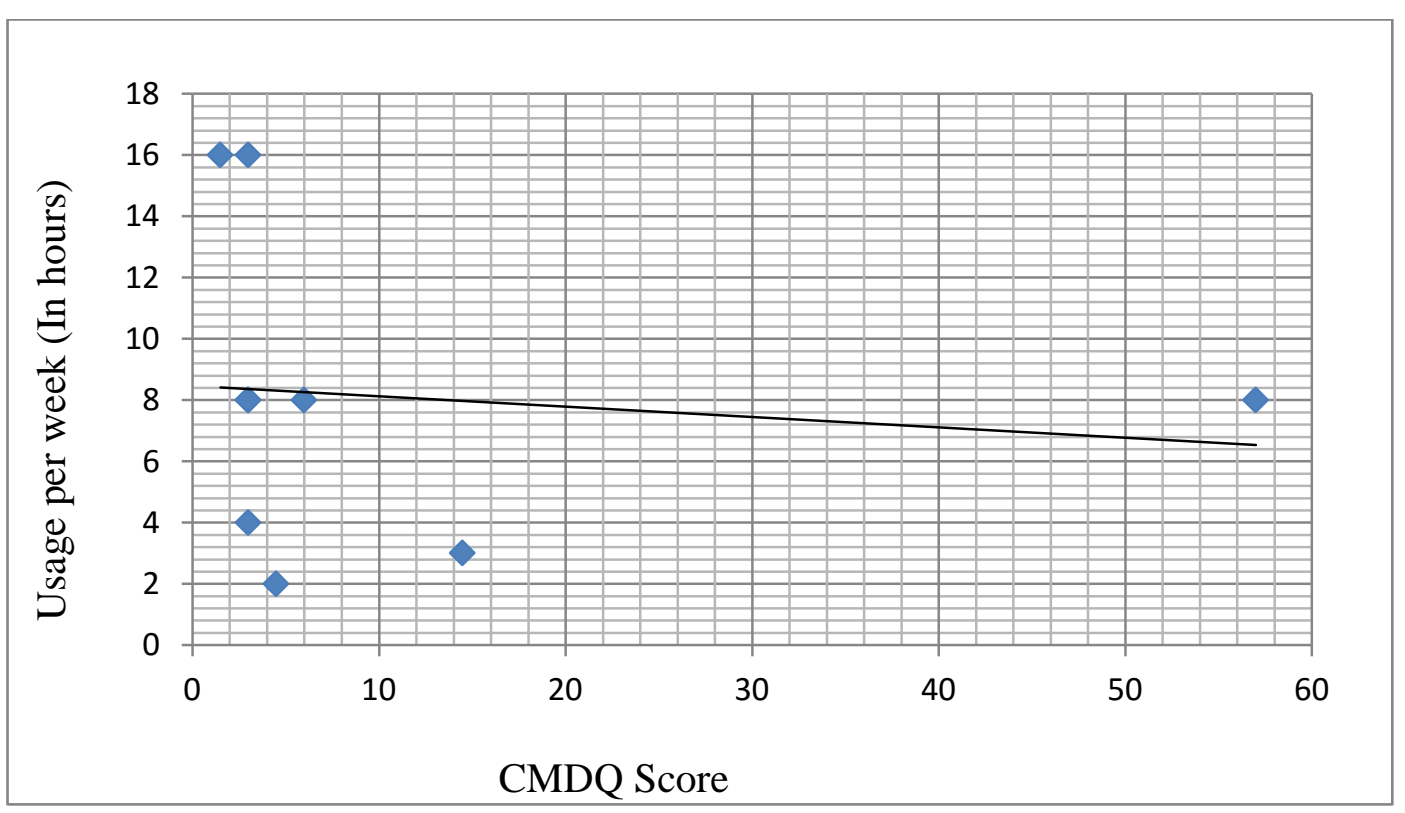

Figure 2: Correlation between usage of instrument per week (in hours) \& CMDQ score 


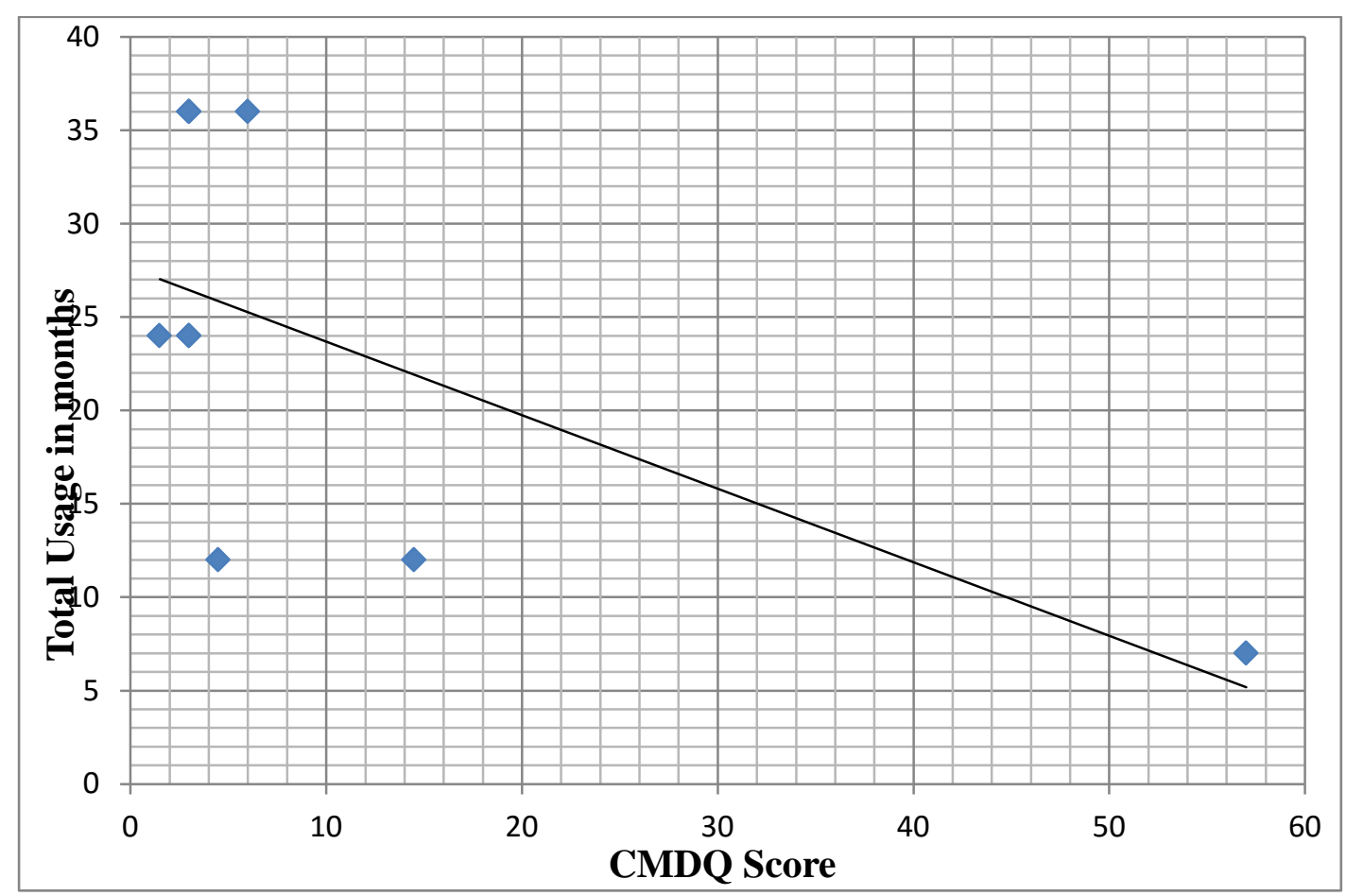

Figure 3: Correlation between total period of usage of the instrument (in months) \&CMDQ score

\section{DISCUSSION}

The study aimed to find the prevalence of playing related musculoskeletal disorders in musicians in Ahmedabad community. In this study, $18 \%$ of PRMD prevalence was found. Also a moderate negative correlation between severity of musculoskeletal symptoms and usage of instruments per week and duration of playing was seen.

A review by Zara $C$ et al found the best estimates of PRMD prevalence were derived from three studies which were $39 \%$ and $47 \%$ in adults and $17 \%$ in secondary school music students respectively.[2] Brandfonbrener et al reported that HMS (hypermobility syndrome) is more common in musicians ( $35 \%$ in women and $17 \%$ in men) compared to non-musicians.[5]Altenmüller et al found the prevalence of focal dystonia in professional musicians is only $1 \%$.[6]

In this study 5 out of 9 (55\%) musicians had neck pain. Fishbein et al found the prevalence of neck pain in $34 \%$ musicians. [8]

In this study 3 out of 9 (33\%) musicians had forearm pain, seven out of nine $(77 \%)$ musicians had wrist pain, 1 out of 9 had upper back pain(11\%) and 1 out of 9 had leg pain(11\%). Shinichi $F$ et al found the finger/hand had the highest rate of PRMDs, followed by the forearm and shoulder.[9] Parry CB et al showed that only $12 \%$ of 507 musicians had a particular diagnosis had tenosynovitis. [4] Charness et al reported ulnar neuropathy was diagnosed in $40 \%$ cases of occupational cramp in musicians. [7,8]However the cause for pain was not evaluated in this study.

In this study, prevalence among music students was overall $18 \%$. Of these $33 \%$ keyboard players and 66\%guitar (string) players were found to have PRMD. Manchester \&Flieder et al found injury incidence rate of performance-related hand problems among music students $(n=114$, median age: 20 ) over 3 years was overall $8.5 \%$, keyboard players $10.4 \%$, string players $9.6 \%$, wind players $2.7 \%$, male $6.1 \%$, female $10.9 \%$.[10]

Norris \& Dommerholt et al suggested ergonomics for string players in the form oforthoses: custom moulding of shoulder and chin rests, tailpiece centred chin rest; instrument supports: "Voelkow" rest for violin, angled end pin for cello; instrument modifications: viola, guitar; and adaptation of size of instrument to anatomical features of the musician.[11]

Zaza \& Farewell et al suggested recommendations on practice habits for musicians which were musical "neuro-muscular" warm-up; stretches can be used as a cool-down, but need to be appropriately performed; inclusion of 10 minute breaks every 20-30 minutes during practice sessions; pacing: gradual increase of practice before concerts, recitals, competitions; variety of content of the practice session: styles of music, 
types of exercises; cognitive rehearsal: away from the instrument; body awareness and control techniques: alexander, Feldenkreis, yoga; stress and anxiety management.[12,13]

\section{CONCLUSION}

$18 \%$ musicians were found to have playing related musculoskeletal disorders. Moderate correlation was found between usage of instrument per week (in hours) \& CMDQ score and between total period of usage of the instrument (in months) \& CMDQ score.

\section{REFERENCES}

1. Berque P. Musculoskeletal disorders affecting musicians and considerations for prevention. Available from: http://www.musicianshealth.co.uk/musiciansmusc uloskeletaldisorders.pdf

2. Zara C. Playing related musculoskeletal disorders in musicians: a systematic review of incidence and prevalence, CMAJ. 1998 Apr 21; 158(8): 1019-1025.

3. Lee HS, Park HY, Yoon JO, Kim JS, Chun JM, Aminata IW, Cho WJ, Jeon IH. Musicians' Medicine: Musculoskeletal problems in string players. Clinics in orthopedic Surgery. 2013 Sep; 5(3): 155-160.

4. WILLIAMON A. Managing the physical demands of musical performance. Musical ExcellenceStrategies and Techniques to Enhance Performance. 2004 Jun 17;41-60. Available from: http://dx.doi.org/10.1093/acprof:oso/9780198525 356.003.0003.

5. Brandfonbrener AG. Joint laxity and arm pain in a large clinical sample of musicians. Medical Problems of Performing Artists. September 2002;17(3):113-115. Available from: https://www.sciandmed.com/mppa/journalviewer. aspx ?issue $=1083 \&$ article $=931$

6. Altenmüller E. Focal dystonia: advances in brain imaging and understanding of fine motor control in musicians. Hand Clinics. 2003 Aug;19(3):523-38.
Available from: http://dx.doi.org/10.1016/s07490712(03)00043-x

7. Charness ME, Ross MH, Shefner JM. Ulnar neuropathy and dystonic flexion of the fourth and fifth digits: Clinical correlation in musicians. Muscle Nerve. 1996 Apr;19(4):431-7. Available from: http://dx.doi.org/10.1002/mus.880190403

8. Fishbein $M$, Middlestadt SE, Ottati V, Straus S, Ellis A. Medical Problems among ICSOM Musicians: Overview of a National Survey. Medical Problems of Performing Artists. 1988;3(1):1-8. Available from: https://www.sciandmed.com/mppa/journalviewer. aspx? issue $=1145 \&$ article $=1451$

9. Furuya $\mathrm{S}$, Nakahara $\mathrm{H}$, Aoki $\mathrm{T}$, Kinoshita $\mathrm{H}$. Prevalence and causal factors of playing-related musculoskeletal disorders of the upper extremity and trunk among japanese pianists and piano students. Medical Problems of Performing Artists.2006 september;21(3):112. Available from: https://www.sciandmed.com/mppa/journalviewer. aspx? issue $=1168 \&$ article $=1669$

10. Manchester RA, Flieder D. Further observations on the epidemiology of hand injuries in music students. Medical Problems of Performing Artists. 1991;6(1):11-14. Available from: https://www.sciandmed.com/mppa/journalviewer. aspx? issue $=1133 \&$ article $=1345$

11. Norris RN. Applied Ergonomics: Aptive equipment and instrument modification for musicians. MD Med J, 1993, Mar;42(3):271-5.

12. Zaza C. Research-based prevention for musicians. Medical problems of performing artists. March 1994;9(1):3-6. Available from: https://www.sciandmed.com/mppa/journalviewer. aspx ?issue $=1121 \&$ article $=1246$

13. Zaza C, Farewell VT. Musicians' playing-related musculoskeletal disorders: An examination of risk factors. American Journal of Industrial Medicine. September 1997;32(3):292-300. 